\title{
Management of bronchiectasis
}

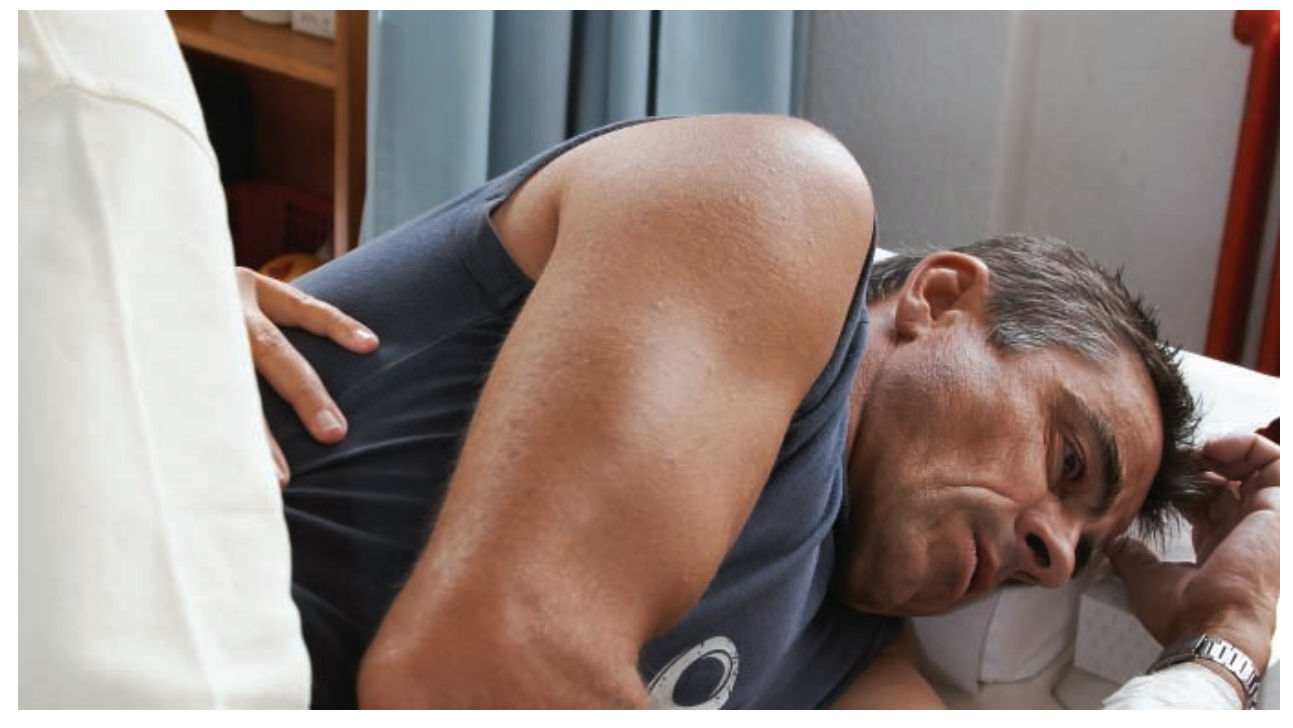

P.T. King ${ }^{1}$

E. Daviskas ${ }^{2}$

${ }^{1}$ Dept of Respiratory and Sleep Medicine/Dept of Medicine, Monash Medical Centre, Melbourne, and ${ }^{2}$ Dept of Respiratory and Sleep Medicine, Royal Prince Alfred Hospital, Sydney, Australia.

\section{Correspondence}

P.T. King

Dept of Respiratory and Sleep

Medicine

Monash Medical Centre

246 Clayton Road

Clayton

Melbourne

Australia

Paul.king@med.monash.edu.au

\section{Provenance}

Commissioned article, peer reviewed.

\section{Educational aims}

1 To describe the specific current and potential future treatments for bronchiectasis.

1 To describe a potential management plan for the treatment of bronchiectasis.

\section{Summary}

Non-cystic fibrosis (non-CF) bronchiectasis remains a common and difficult respiratory condition to manage. Patients with bronchiectasis generally tend to have persistent symptoms and require long-term medical treatment. There are a large number of treatment options available for the management of bronchiectasis. However only a limited number of trials to assess efficacy of treatment have been conducted. These studies have generally included fewer than 20 patients and very few of them have been randomised or blinded. As such, the management of bronchiectasis is not clearly defined. This review will discuss the specific treatments available to treat patients with non-CF bronchiectasis and how they could be integrated into a specific management plan.

Specific treatments for bronchiectasis can

be considered under the broad categories of: 1) antibiotics; 2) enhancement of mucociliary and cough clearance; and 3) other therapies. There is no clearly validated treatment plan for bronchiectasis but important aspects include: 1) management of exacerbations; 2) management of clinically stable disease; 3) regular follow-up; and 4) identification of the subject with severe or rapidly deteriorating disease.

\section{Competing interests}

E. Daviskas is an employee of the South West Sydney Area Health Service that owns the patent relating to the use of mannitol for enhancing clearance of secretions and may benefit from royalties in the future. E. Daviskas owns self-funded shares in Pharmaxis Ltd and, in her capacity as an employee of the SSWAHS, consults for Pharmaxis Ltd. 


\section{Specific treatments}

\section{Antibiotics}

The use of antibiotics in bronchiectasis is complicated. There is a range of pathogens involved, resistant organisms are common and often it is not possible to isolate any pathogens, despite the presence of purulent sputum $[1,2]$. In addition, patients may be colonised simultaneously with multiple pathogens and the presence of biofilms, particularly those formed by Pseudomonas aeruginosa, may block the effect of antibiotics. There is a change in bacteria isolated in different stages of disease, with nontypeable Haemophilus influenzae being common in less severe disease and $P$. aeruginosa being prevalent in subjects with more advanced disease [2]. Examining multiple sputum samples, if necessary by bronchoscopy, to obtain a microbiological diagnosis is very useful. Antibiotics may be used both for treating exacerbations and as maintenance therapy.

The main indication for the use of antibiotics is for exacerbations, in which antibiotics have been shown to improve quality of life and systemic symptoms and decrease to airway inflammation. Murrar et al. [3], in a follow-up study of patients with a total of 32 exacerbations, found that 14 days of intravenous antibiotics improved symptoms, inflammatory markers, sputum and quality of life (but not lung function). Generally, patients are treated initially with one or two standard oral antibiotics for 10-14 days. The prescription of a longer course is common practice but there is no clear evidence that this is beneficial. Intravenous antibiotics should be used for patients who are severely unwell or who fail to respond to oral antibiotics.

Antibiotics may be used as maintenance therapy. The use of continuous or monthly antibiotics may have mild benefits including reduction in symptoms, improved sputum parameters, reduction in lung inflammation and improvement in lung function [4].

Tetracyclines have been used for purulent bronchitis/bronchiectasis but evidence for their effectiveness is inconclusive [5]. A 12-month randomised trial of tetracycline improved clinical status. The main concern with the prolonged use of antibiotics is the development of bacterial resistance. The use of oral quinolones to treat Pseudomonas infection is associated with a high rate of resistance after one or two courses [6].

Antibiotics may also be given via an inha lational route. Nebulised tobramycin in some patients caused significant clinical improvement and a high rate of pathogen clearance [7]. There was a reasonably high incidence of sideeffects. Inhaled gentamicin given for 3 days decreased sputum production and improved lung function [8].

There has recently been interest in the use of macrolide antibiotics for the treatment of bronchiectasis. The macrolides also have immunomodulatory properties that may be useful in this context [9]. Several small trials have assessed the use of microlides in non-CF bronchiectasis. These trials, which have been up to 6 months in duration, have reported promising results with reduced exacerbations and sputum and improved symptoms [10]. There is some concern that they may induce resistance in nontuberculous mycobacteria.

\section{Enhancement of mucociliary and cough clearance}

Effective clearance of excessive mucus from the airways is very important as it can potentially break the vicious cycle of the disease by minimising: 1) stagnation of mucus; 2) mucus plug formation; 3) bacterial load; 4) inflammation; and 5) recurrent infections. Patients have chronic cough and recurrent infections mainly because the mucociliary escalator fails to clear the excessive load of mucus. However, the cough is not very effective in clearing mucus because the mucus is dehydrated, viscoelastic and sticky. In addition, cough requires high expiratory flow to be effective and can only have an effect, if at all, in the large airways.

Recent approaches to the treatment of mucociliary dysfunction aim to increase hydration at the airway surface, improve the physical properties of mucus and, thus, facilitate its clearance by ciliary and cough action [11, 12]. Osmotic agents, such as mannitol and hypertonic saline, given by inhalation, increase the osmolarity of the airway surface fluid and create a driving force for water to move quickly into the airway lumen. They have the capacity to increase or restore airway hydration, alter the physical properties of mucus [13, 14] and, thus, improve mucociliary and cough clearance. In addition, osmotic agents have the potential to reduce the viscoelastic properties of mucus by breaking some of the bonds the mucins form, thereby reducing their entanglements [15]. Unlike the classical mucolytics, e.g. N-acetycysteine and bromhexine, osmotic agents do not denature the mucus by breaking the disulphide bonds between mucins [15]. Osmotic stimuli are a very promising treatment avenue in patients with bronchiectasis [16]. 


\section{Mannitol (potential future treatment)}

Mannitol is a naturally occurring sugar alcohol that is not readily metabolised and does not cross membranes, and so its effect can be sustained in the airways for hours. It is delivered as a dry powder from capsules, conveniently using a dry powder inhaler, with a close to full inspiration at a flow between 60-90 L per min, which most patients can achieve. Studies showed that a single dose of mannitol $(400 \mathrm{mg})$ increases clearance of mucus both acutely and over $24 \mathrm{~h}$ in patients with bronchiectasis $[17,18]$. Clearance of mucus after mannitol inhalation was increased from a mean of $12 \%$ per $h$ at baseline to a mean value of $34 \%$ per $h$ with mannitol, a value similar to that measured in healthy subjects. An important finding was that mucociliary clearance $2 \mathrm{~h}$ after mannitol inhalation was equivalent to that which would normally take $24 \mathrm{~h}$ [18]. Another important finding was that inhalation of mannitol improved clearance from all lung regions, including the peripheral region [18], a finding consistent with the mannitol depositing with a diffuse pattern in the lung $[19,20]$. A recent study showed that the improvement in mucociliary clearance was dose dependent and that the effect of mannitol was enhanced when it was followed by voluntary coughing [21]. It would follow, therefore, that patients may have increased clearance if, within $1 \mathrm{~h}$ after mannitol inhalation, they also undertake some physiotherapy manoeuvres. All the above studies, although they were acute and were carried out in a small number of patients, set the scientific basis for investigating the long-term clinical effect of mannitol in bronchiectasis.

Long-term, randomised placebo-controlled studies showed that effective clearance of mucus with inhaled mannitol, twice daily, over 2 weeks (400 mg) ( $\mathrm{n}=17)$ and over 12 weeks (320 mg) $(n=185)$ improved health-related quality of life, as assessed by the St George's Respiratory Questionnaire, in non-CF bronchiectasis [22, 23]. In addition, patients receiving regular treatment with mannitol over 12 weeks had a longer time free of antibiotic use compared with patients receiving placebo [23]. Currently, multicentre studies are investigating the effect of mannitol on the exacerbation rate over 12 months. Mannitol has the potential to be a future treatment in bronchiectasis.

\section{Hypertonic saline/normal saline}

Hypertonic saline, unlike mannitol, is an ionic substance that is quickly transported across the epithelium, so it may not sustain the osmotic effect for long in patients with non-CF bronchiectasis. It is delivered as a wet aerosol using an ultrasonic or jet nebuliser with tidal breathing and the highest well tolerated concentration is $7 \%$. The effect of hypertonic saline has not been well documented in non-CF bronchiectasis. There is evidence that the combination of hypertonic saline treatment with a physiotherapy technique can acutely improve expectoration of sputum [24]. In a recent long-term study, use of hypertonic or isotonic saline over 12 months, in combination with a physiotherapy technique, improved lung function and health-related quality of life [25]. Thus, hypertonic/isotonic saline may be beneficial in bronchiectasis.

\section{Tolerability and safety of osmotic stimuli in bronchiectasis}

Osmotic stimuli can provoke cough after inhalation but this coughing, as documented in studies using mannitol short- or long-term, has been well tolerated by patients with bronchiectasis [22, 23]. One should keep in mind that bronchiectasis patients rely on cough to clear their secretions, so the cough provoked by osmotic stimuli is synergistic and thus beneficial, as opposed to an unwanted sideeffect. There was no change in the sputum microbiology and there were no adverse effects reported in relation to mannitol in patients receiving mannitol over a maximum of 12 weeks [23]. The longer term (12 months) clinical effect of treatment with mannitol is currently under investigation.

Osmotic stimuli, when inhaled, have the potential to cause airway narrowing in patients with hypersensitive airways [26]. As some patients have asthma in addition to bronchiectasis, a bronchial provocation test with mannitol or hypertonic saline is highly recommended before starting treatment with mannitol or hypertonic saline. Patients with bronchiectasis who have a significant fall in forced expiratory volume in $1 \mathrm{~s}(>15 \%)$ from baseline value in response to mannitol or hypertonic saline should not be deprived of treatment for clearing mucus. They may simply need to be treated for their asthma before commencing on mannitol or hypertonic saline treatment. In most bronchiectasis patients, asthma appears to be mild and acute protection against airway narrowing in response to mannitol has been achieved with premedication with nedocromil sodium [17, 18], sodium cromoglycate and eformoterol [27]. 


\section{Educational questions}

1) Which of the following is true?

a) Improving clearance of mucus in the small airways is not important

b) Effective clearance of mucus with osmotic stimuli does not improves the quality of life in bronchiectasis

c) rhDNase is recommended in bronchiectasis

d) Physiotherapy techniques combined with osmotic stimuli can contribute to effective airway clearance and improve quality of life

2) Surgery for bronchiectasis is indicated for:

a) Generalised disease b) Infection with $P$. aeruginosa

c) Mild localised disease

d) Severe localised disease

3) Exacerbations of bronchiectasis:

a) Are a well-defined and understood process

b) May require parenteral antibiotics

c) Should be treated with corticosteroids

d) Should be treated with inhaled antibiotics

\section{rhDNase}

Recombinant human DNase (rhDNase, Pulmozyme, dornase alfa) has the potential to change the rheology of purulent mucus by degrading the DNA filaments, and thus to improve clearance of mucus. However, rhDNase has not been shown to have any beneficial effect in patients with non-CF bronchiectasis and potentially it can be harmful $[28,29]$. In a large multicentre clinical trial, patients with bronchiectasis receiving rhDNase had higher exacerbation and hospitalisation rates and more rapid decline in lung function compared with the placebo group [29].

\section{Physiotherapy and pulmonary rehabilitation} Chest physiotherapy has been used for many years and aims to improve clearance of mucus from the airways by loosening and mobilising the mucus and facilitating its expectoration. Several techniques are available, such as postural drainage, active cycle of breathing technique, positive expiratory pressure (PEP), oscillatory PEP devices and high-frequency chest wall percussion, but few controlled studies have documented a clinical benefit in bronchiecta sis. However, recent studies provide evidence that regular physiotherapy improves expectoration of sputum and quality of life $[30,31]$.

There are no controlled studies showing the long-term clinical effect of combining physiotherapy with other treatments such as osmotic stimuli. Combination of osmotic stimuli with a physiotherapy technique may prove to be the most efficient method in maximising clearance of excessive mucus from the airways.

A randomised trial demonstrated that pulmonary rehabilitation improved exercise tolerance in bronchiectasis [32].

\section{Other treatment modalities Bronchodilators}

A large proportion of subjects with bronchiectasis have airway reversibility. Whether this is due to asthma or bronchial hyperreactivity with infection has not been established. Asthma-like symptoms are associated with accelerated decline in lung function [33]. It is therefore appropriate to use bronchodilators for the treatment of bronchiectasis. Long-acting $\beta_{2}$-agonists have been used for patients with bronchiectasis, though a Cochrane review found there were no randomised trials to support its use [34].

\section{Corticosteroids}

The use of corticosteroids has not been clearly defined in bronchiectasis. Subjects with co-existent asthma should be treated with corticosteroids in accordance with established guidelines. However, it may be difficult to separate out asthma and bronchiectasis. Corticosteroids, owing to their immunosuppressive properties, have the potential to make underlying infection worse.

Oral corticosteroids appear to slow progression of lung disease in CF, but have a high incidence of side-ffects [35]. There are no randomised trials assessing the use of oral steroids in bronchiectasis.

The use of inhaled corticosteroids (ICS) in bronchiectasis has been reviewed recently [36]. The role of ICS has not been established in bronchiectasis, although benefits have been described, including a trend towards improved lung function and decreased sputum. The only study of more than 6 months' duration failed to show any improvement in outcomes. Ho et al. [37] showed an improvement in a subgroup of patients with $P$. aeruginosa infection. Withdrawal of ICS after a 12-week course produced increased bronchial hyperreactivity and decreased neutrophil apoptosis but no other changes [38].

\section{Surgery}

Surgery with lobectomy/pneumonectomy was previously widely used for the treatment of bronchiectasis. However, with improved medical therapy (particularly with antibiotics) this is not commonly done.

Surgery is now generally offered to selected patients who have localised disease and severe symptoms. Two studies have reported that $80 \%$ of patients improve postoperatively and there was a low incidence of mortality and morbidity $[39,40]$. However, concerns have been raised that there are risks and mortality with such surgery. Lobectomy may also be done thoracoscopically [41]. Factors that may be associated with a worse outcome include incomplete resection, post-tuberculous lung damage and worse lung function. Transplant is also performed in non-CF bronchiectasis but there are no clearly defined outcome figures.

\section{Vaccination}

Standard guidelines for the treatment of chronic obstructive pulmonary disease (COPD) advise that vaccination should be given regularly. Influenza vaccine may reduce exacerbations of COPD [42]. Vaccination is also recommended in bronchiectasis but the benefit has not been definitively established. Patients should probably receive a yearly influenza vaccine and a 5-yearly pneumococcal vaccine. 
Management of upper respiratory tract disease Upper respiratory tract disease, ranging from rhinitis to severe pansinusitis, is a very common manifestation of bronchiectasis [43], particularly in those with childhood-onset disease [44]. Unfortunately this is a difficult problem that is often poorly responsive to therapy.

Saline irrigation may help to clear secretions and can be done using a spray or by more vigorous washing. This has been shown to be helpful and may be given multiple times a day. The saline may be isotonic or hypertonic. Hypertonic saline may be more effective but has more side-effects. Other topical agents, such as decongestants and $N$-acetylcysteine, are used but there is little published data on effectiveness. Antihistamines may be beneficial in patients with associated atopy.

Topical steroids are commonly used for the treatment of rhinosinusitis and they are effective in nasal polyps, including in CF. Steroids do have a potential problem of making infection worse due to their immunosuppressive properties.

Antibiotics are frequently prescribed for the treatment of upper respiratory tract disease. The macrolides in particular may have a particular role and their use has produced a dramatic improvement in Japanese subjects with diffuse panbronchiolitis [10] and sinusitis. Part of this effect may be through their immunomodulatory action. There appear to have been no published trials on the use of antibiotics for the treatment of rhinosinusitis in bronchiectasis.

Endoscopic surgery may be performed particularly for localised obstruction. Sinus surgery has been shown to improve symptoms in CF but there is a lack of long-term data.

\section{Treatment of associated conditions}

Smoking is associated with worse lung function in bronchiectasis and all patients should be encouraged to stop smoking $[45,46]$. Exposure to biomass fuel combustion products increases the incidence of lower respiratory tract infection [47] and should also be avoided.

Hypogammaglobulinaemia is an important aetiological cause and all subjects should be offered replacement immunoglobulin therapy. The role of immunoglobulin subclass deficiency in bronchiectasis is less well defined and the role of replacement therapy is controversial.

Oesophageal reflux may have a role in bronchiectasis and should be treated appropriately.

\section{Nutrition}

The immune system is impaired by nutritional deficiency with a resultant increase in respiratory

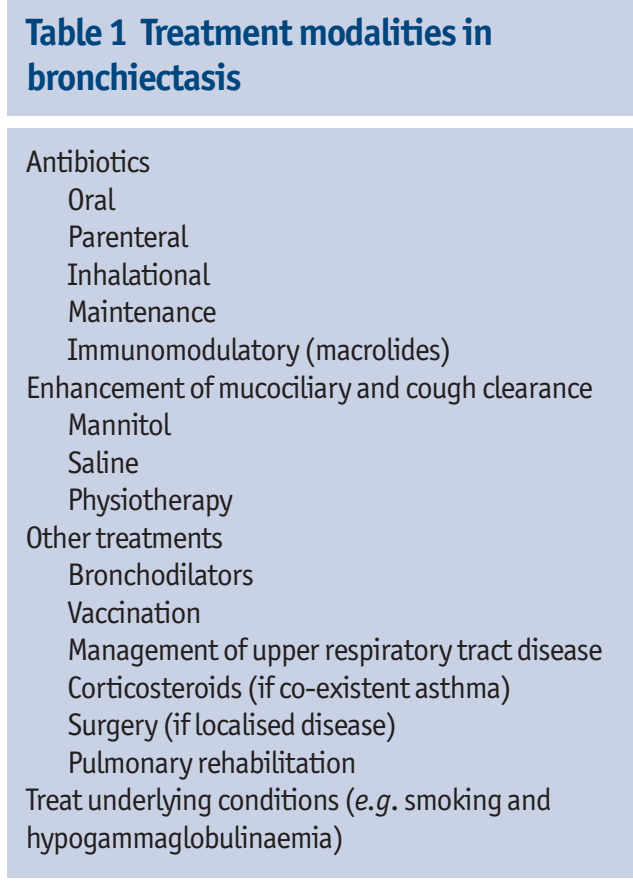

infections. A low body mass index (BMI) is a marker of poor outcome in COPD [48]. Adequate nutrition reduces lung infections and may also improve lung function. This may be particularly important in children. Nutrition is one of the key issues in the management of CF and, while the literature in bronchiectasis is minimal, subjects with a low BMI should probably have a nutrient assessment and be referred to a dietician.

\section{Management plan}

The development of a management plan may be of significant benefit to patients. Early institution of treatment is thought to be beneficial in chronic inflammatory airway diseases, such as asthma and COPD. In primary ciliary dyskinesia, early initiation of appropriate antibiotic and sputum clearance techniques prevents decline in lung function and clinical status [49].

Unlike other respiratory diseases, such as as-thma or COPD, there is no accepted management plan for bronchiectasis. Bronchiectasis is a heterogeneous condition with a great range in clinical severity, and management needs to be tailored for the individual patient. We suggest some important steps which may help to guide treatment. Table 1 lists treatment modalities in bronchiectasis.

\section{Initial patient assessment}

All subjects should have a comprehensive history and physical examination. Bronchiectasis is usually diagnosed by high-resolution computed 


\section{Table 2 Investigations on initial assessment}

HRCT to diagnose bronchiectasis

Lung function

Lung volumes and bronchodilator response

Sputum analysis

Multiple specimens

Mycobacterial analysis

Consider bronchoscopy

Screen for underlying cause

Full blood examination

Immunoglobulins

ABPA

Other tests could include: HIV, exhaled nitrous oxide/ciliary function, sweat

Rhinosinusitis test/CF mutation analysis

Radiograph of sinuses (plain or computed tomography)

Otolaryngotologist review

HRCT: high-resolution computed tomography; ABPA: allergic bronchopulmonary aspergillosis.

Figure 1

Suggested management plan for bronchiectasis patients. tomography scanning and all subjects should have at least one baseline scan. Patients require baseline lung function assessment with spirometry (including pre- and postbronchodilator) and, ideally, lung diffusing capacity. Bronchiectasis is associated with immune deficiency and screening blood tests for immune function should be performed. The blood tests should include a full blood examination, immunoglobulins and

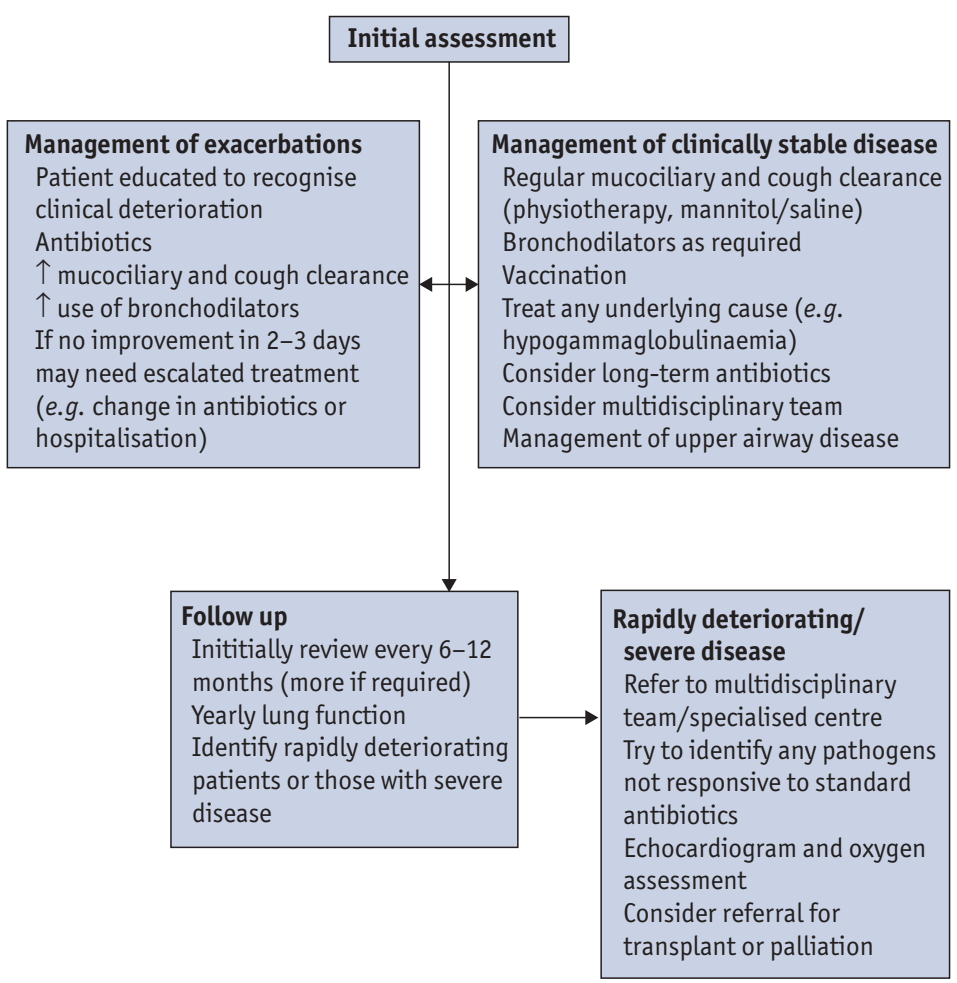

Aspergillus serology, as these tests have a direct effect on treatment.

It is also very important to obtain microbiological evaluation of the airways. Multiple sputum samples should be sent for standard microscopy, culture and sensitivity, including fungal and mycobacterial assessment. Unfortunately, in a large proportion of patients, despite the presence of purulent sputum no pathogen can be isolated. In this circumstance bronchoscopy should be considered (which also may not yield any identifiable pathogen [1]). Table 2 lists investigations that are appropriate for the initial assessment.

\section{Management of exacerbations}

Exacerbations are an important clinical feature of bronchiectasis, although there is no generally accepted definition [50]. Treatment of exacerbations is important for preventing decline in lung function and quality of life. There is very limited literature describing the specific management of exacerbations of bronchiectasis.

Treatment usually consists of appropriate antibiotics and increased airway clearance. One systematic review found that antibiotics were beneficial. Quinolones have been found to be superior to $\beta$-lactam antibiotics in acute exacerbations, although there are concerns about antibiotic resistance. Noninvasive ventilation (NIV) may be used in subjects with hypercapnic respiratory failure.

Action plans in which patients initiate treatment in an exacerbation have been well established in asthma. Early initiation of treatment may be helpful in bronchiectasis. In this circumstance patients may be prescribed antibiotics to keep at home and commence as soon as they think they are developing an exacerbation.

\section{Management of clinically stable disease}

Subjects with bronchiectasis should all be reviewed regularly, especially in the first several years after diagnosis. Some patients develop rapidly progressive disease and it is important to identify these subjects early. This can be done by regular clinical assessment and yearly lung function tests.

Maintenance therapy consists of regular airway clearance, bronchodilator use, vaccination, exercise and minimising aggravating factors. Treatment of specific conditions, such as reflux and hypogammaglobulinaemia, may also be helpful. 
The use of a multidisciplinary team approach, as is used in CF, may be appropriate, particularly for subjects with more severe disease.

Another important aspect of treatment is self-management of the disease by the patient. As bronchiectasis is a heterogeneous condition with a variety of generally not-well-validated treatments, successful management is dependent on the patient determining what works for them [51]. This applies both for exacerbations and in clinically stable disease.

\section{Management of the patient with severe disease}

The introduction of antibiotics has vastly improved outcome in bronchiectasis, but this condition still has a level of mortality and there is a significant proportion of patients with severe disease. There is also a small proportion of subjects who develop rapid decline in lung function. It is important to identify these patients as there are some important treatment considerations in this cohort.

It is very important to determine whether the patient has been colonised by a resistant pathogen, such as $P$. aeruginosa. Significant efforts should be made to analyse multiple sputum samples including consideration for bronchoscopy. Mycobacterial analysis should be performed as well.

Subjects should have an oxygen assessment and supplemental therapy should be given if indicated. Assessment of cardiac (particularly right heart) function should be done by transthoracic echocardiogram. NIV may be beneficial in acute hypercapnic respiratory failure in CF [52] and bronchiectasis [53]. The role of NIV for chronic respiratory failure has not been defined. Long-term humidification of air may reduce exacerbations, and improve lung function and quality of life [54]. For subjects with the most severe disease, consideration of referral to a transplant service or, alternatively, palliative care may be appropriate. Figure 1 gives a suggested management programme for bronchiectasis

\section{Conclusion}

Bronchiectasis remains an important cause of respiratory disease. Patients generally have persistent symptoms and tend to lose lung function gradually. The treatments available for this condition have generally not been well validated. Our experience is that appropriate institution of the available treatment modalities and careful followup does help control clinical symptoms and may alter the course of the disease. Better clinical studies are needed to establish the most effective ways of managing bronchiectasis.

\section{Acknowledgements}

The authors would like to thank P. Holmes (Monash Medical Centre, Melbourne, Australia) and S. Anderson (Royal Prince Alfred Hospital, Sydney, Australia) for their help with this work.

\section{References}

1. Angrill J, Agusti C, de Celis R, et al. Bacterial colonisation in patients with bronchiectasis: microbiological pattern and risk factors. Thorax 2002; 57: 15-19.

2. King PT, Holdsworth SR, Freezer NJ, et al. Microbiologic follow-up study in adult bronchiectasis. Respir Med 2007; 101: 1633-1638.

3. Murray MP, Turnbull K, Macquarrie S, et al. Assessing response to treatment of exacerbations of bronchiectasis in adults. Eur Respir J 2009; 33: 312-318.

4. Evans DJ, Bara AI, Greenstone M. Prolonged antibiotics for purulent bronchiectasis. Cochrane Database Syst Rev 2003; 4: CD001392.

5. Pines A. The tetracyclines in purulent exacerbations of chronic bronchitis. J Antimicrob Chemother 1982; 9: 333-335.

6. Rayner CF, Tillotson G, Cole PJ, et al. Efficacy and safety of long-term ciprofloxacin in the management of severe bronchiectasis. J Antimicrob Chemother 1994; 34: 149-156.

7. Lobue PA. Inhaled tobramycin: not just for cystic fibrosis anymore? Chest 2005; 127: 1098-1101.

8. Lin $\mathrm{HC}$, Cheng $\mathrm{HF}$, Wang $\mathrm{CH}$, et al. Inhaled gentamicin reduces airway neutrophil activity and mucus secretion in bronchiectasis. Am J Respir Crit Care Med 1997; 155: 2024-2029.

9. Bush A, Rubin BK. Macrolides as biological response modifiers in cystic fibrosis and bronchiectasis. Semin Respir Crit Care Med 2003; 24: 737-748.

10. King P. Is there a role for inhaled corticosteroids and macrolide therapy in bronchiectasis? Drugs 2007; 67: 965-974.

11. Hirsh AJ. Altering airway surface liquid volume: inhalation therapy with amiloride and hyperosmotic agents. Adv Drug Deliv Rev 2002; 54: 1445-1462.

12. Tarran R. Regulation of airway surface liquid volume and mucus transport by active ion transport. Proc Am Thorac Soc 2004; 1: 42-46.

13. Daviskas E, Anderson SD, Gomes K, et al. Inhaled mannitol for the treatment of mucociliary dysfunction in patients with bronchiectasis: effect on lung function, health status and sputum. Respirology 2005; 10: 46-56.

14. Daviskas E, Anderson SD, Young IH. Effect of mannitol and repetitive coughing on the sputum properties in bronchiectasis. Respir Med 2010; 104: 371-377.

15. King M, Rubin BK. Pharmacological approaches to discovery and development of new mucolytic agents. Adv Drug Deliv Rev 2002; 54: 1475-1490.

16. Wills P, Greenstone M. Inhaled hyperosmolar agents for bronchiectasis. Cochrane Database Syst Rev 2006; 2: CD002996. 
17. Daviskas $\mathrm{E}$, Anderson SD, Eberl S, et al. Inhalation of dry powder mannitol improves clearance of mucus in patients with bronchiectasis. Am J Respir Crit Care Med 1999; 159: 1843-1848.

18. Daviskas E, Anderson SD, Eberl S, et al. The 24-h effect of mannitol on the clearance of mucus in patients with bronchiectasis. Chest 2001; 119: 414-421.

19. Glover W, Chan H-K, Eberl S, et al. Lung deposition of mannitol powder aerosol in healthy subjects. J Aerosol Med 2006; 19: 522-532.

20. Glover W, Chan HK, Eberl S, et al. Effect of particle size of dry powder mannitol on the lung deposition in healthy volunteers. Int J Pharm 2008; 349: 314-322.

21. Daviskas $E$, Anderson SD, Eberl $S$, et al. Effect of increasing doses of mannitol on mucus clearance in patients with bronchiectasis. Eur Respir J 2008; 31: 765-772.

22. Daviskas E, Turton JA, Anderson SD, et al. A placebo controlled trial with inhaled mannitol improves health related quality of life in patients with bronchiectasis. Eur Respir J 2004; 24: Suppl. 48, 707S.

23. Bilton D, Daviskas $E$, Jaques $A$, et al. A randomised, placebo-controlled trial of inhaled mannitol in patients with bronchiectasis. Eur Respir J 2008; 32: Suppl. 52, 85 S.

24. Kellett F, Redfern J, Niven RM. Evaluation of nebulised hypertonic saline (7\%) as an adjunct to physiotherapy in patients with stable bronchiectasis. Respir Med 2005; 99: 27-31.

25. Nicholson C, Stirling R, Borg B, et al. The long term effect of hypertonic saline (6\%) in non-cystic fibrosis bronchiectasis. Respirology 2010; 15: Suppl. 1, A28.

26. Brannan JD, Anderson SD, Perry CP, et al. The safety and efficacy of inhaled dry powder mannitol as a bronchial provocation test for airway hyperresponsiveness: a phase 3 comparison study with hypertonic (4.5\%) saline. Respir Res 2005; 6: 144.

27. Briffa P, Anderson SD, Burton DL, et al. Prevention of airway narrowing following mannitol inhalation in subjects with bronchiectasis. Respirology 2009; 14: Suppl. 1, A3.

28. Wills PJ, Wodehouse T, Corkery K, et al. Short-term recombinant human DNase in bronchiectasis. Effect on clinical state and in vitro sputum transportability. Am J Respir Crit Care Med 1996; 154: 413-417.

29. O'Donnell AE, Barker AF, Ilowite JS, et al. Treatment of idiopathic bronchiectasis with aerosolized recombinant human DNase I. rhDNase Study Group. Chest 1998; 113: 1329-1334.

30. Mutalithas K, Watkin G, Willig B, et al. Improvement in health status following bronchopulmonary hygiene physical therapy in patients with bronchiectasis. Respir Med 2008; 102: 1140-1144.

31. Murray MP, Pentland JL, Hill AT. A randomised crossover trial of chest physiotherapy in non-cystic fibrosis bronchiectasis. Eur Respir J 2009; 34: 1086-1092.

32. Newall C, Stockley RA, Hill SL. Exercise training and inspiratory muscle training in patients with bronchiectasis. Thorax 2005; 60: 943-948.

33. Keistinen T, Saynajakangas 0, Tuuponen T, et al. Bronchiectasis: an orphan disease with a poorly-understood prognosis. Eur Respir J 1997; 10: 2784-2787.

34. Sheikh A, Nolan D, Greenstone M. Long-acting $\beta_{2}$-agonists for bronchiectasis. Cochrane Database Syst Rev 2001; 4: CD002155.

35. Rosen MJ. Chronic cough due to bronchiectasis: ACCP evidence-based clinical practice guidelines. Chest 2006; 129: Suppl. 1, 122S-131S.

36. Kapur N, Bell S, Kolbe J, et al. Inhaled steroids for bronchiectasis. Cochrane Database Syst Rev 2009; 1: CD000996.

37. Ho PL, Chan KN, Ip MS, et al. The effect of Pseudomonas aeruginosa infection on clinical parameters in steady-state bronchiectasis. Chest 1998; 114: 1594-1598.

38. Guran T, Ersu R, Karadag B, et al. Withdrawal of inhaled steroids in children with non-cystic fibrosis bronchiectasis. J Clin Pharm Ther 2008; 33: 603-611.

39. Agasthian T, Deschamps C, Trastek VF, et al. Surgical management of bronchiectasis. Ann Thorac Surg 1996; 62: 976-978.

40. Balkanli K, Genc 0 , Dakak M, et al. Surgical management of bronchiectasis: analysis and short-term results in 238 patients. Eur J Cardiothorac Surg 2003; 24: 699-702.

41. Rothenberg SS. First decade's experience with thoracoscopic lobectomy in infants and children. J Pediatr Surg 2008; 43: 40-44.

42. Poole PJ, Chacko E, Wood-Baker RW, et al. Influenza vaccine for patients with chronic obstructive pulmonary disease. Cochrane Database Syst Rev 2000; 4: CD002733.

43. Loebinger MR, Bilton D, Wilson R. Upper airway 2: bronchiectasis, cystic fibrosis and sinusitis. Thorax 2009; 64: 1096-1101.

44. King PT, Holdsworth SR, Farmer MW, et al. Phenotypes of bronchiectasis; onset of productive cough in childhood and adulthood. COPD 2009; 6: 130-136.

45. Nicotra MB, Rivera M, Dale AM, et al.. Clinical, pathophysiologic, and microbiologic characterization of bronchiectasis in an aging cohort. Chest 1995; 108: 955-961.

46. King PT, Holdsworth S, Farmer MW, et al. Phenotypes of bronchiectasis: onset of productive cough in childhood and adulthood. COPD 2009; 6: 130-136.

47. Ezzati M, Kammen D. Indoor air pollution from biomass combustion and acute respiratory infections in Kenya: an exposure-response study. Lancet 2001; 358: 619-624.

48. Celli BR, Cote CG, Marin JM, et al. The body-mass index, airflow obstruction, dyspnea, and exercise capacity index in chronic obstructive pulmonary disease. N Engl J Med 2004; 350: 1005-1012.

49. Ellerman A, Bisgaard $\mathrm{H}$. Longitudinal study of lung function in a cohort of primary ciliary dyskinesia. Eur Respir J 1997; 10: 2376-2379.

50. Chang AB, Grimwood K, Maguire G, et al. Management of bronchiectasis and chronic suppurative lung disease in indigenous children and adults from rural and remote Australian communities. Med J Aust 2008; 189: 386-393.

51. Lavery K, 0'Neill B, Elborn JS, et al. Self-management in bronchiectasis: the patients' perspective. Eur Respir J 2007; 29: 541-547.

52. Holland AE, Denehy L, Ntoumenopoulos G, et al. Non-invasive ventilation assists chest physiotherapy in adults with acute exacerbations of cystic fibrosis. Thorax 2003; 58: 880-884.

53. British Thoracic Society Standards of Care Committee. Non-invasive ventilation in acute respiratory failure. Thorax 2002; 57: 192-211.

54. Rea H, McAuley S, Jayaram L,et al.The clinical utility of long-term humidification therapy in chronic airway disease. Respir Med 2010; 104: 525-533. 